\title{
A fatal case of tuberculous meningitis in a child with juvenile idiopathic arthritis: a diagnostic challenge
}

\author{
Zumrut Sahbudak Bal ${ }^{[1]}$, Pinar Yazici ${ }^{[1]}$, Semra Sen ${ }^{[1]}$, Cenk Eraslan ${ }^{[2]}$, \\ Cengiz Cavusoglu ${ }^{[3]}$, Bulent Karapinar ${ }^{[1]}$ and Fadil Vardar ${ }^{[1]}$
}

[1]. Department of Pediatrics, Medical School of Ege University, Bornova, Izmir, Turkey. [2]. Department of Radiology, Medical School of Ege University, Bornova, Izmir, Turkey. [3]. Department of Microbiology and Clinical Microbiology, Medical School of Ege University, Bornova, Izmir, Turkey.

\begin{abstract}
The prognosis of tuberculous meningitis, a rare form of extrapulmonary tuberculosis, depends on the stage of treatment initiation. We report a fatal case of tuberculous meningitis. The patient had received successive tumor necrosis factor (TNF) antagonists and abatacept to treat juvenile idiopathic arthritis, with negative results for polymerase chain reaction and acid-fast bacilli on smear, had normal cerebrospinal fluid (CSF) adenosine deaminase and glucose levels. Six weeks post-admission, the CSF culture demonstrated Mycobacterium tuberculosis. The altered immunological responses caused by anti-TNF treatment made the diagnosis challenging. Clinicians should bear this in mind and, if suspected, treatment should be initiated immediately.
\end{abstract}

Keywords: Tuberculosis. Tumor necrosis factor antagonist. Juvenile idiopathic arthritis.

\section{INTRODUCTION}

Tuberculous meningitis (TBM) accounts for about $1 \%$ of all cases of tuberculosis (TB) and 5\% of all cases of extrapulmonary TB in immunocompetent individuals; patients with TBM have a high mortality rate, and residual neurologic sequelae can occur despite effective treatment $t^{1,2}$. Rheumatic disease itself is associated with a 2-4-fold increase in the risk of TB, even without the use of anti-tumor necrosis factor alpha $(\mathrm{TNF}-\alpha)$ medications ${ }^{3}$. Cases of active tuberculosis associated with the use of therapeutic agents that inhibit TNF- $\alpha$ have been reported worldwide. TNF- $\alpha$ plays a central role in mycobacterial infection and disease. Accordingly, progression of recently acquired TB infection or reactivation of previously acquired infection should be expected with the use of anti-TNF agents ${ }^{4,5}$. Early recognition is important because the clinical outcome depends upon the stage at which therapy is initiated ${ }^{1}$. A recently published review article reported 5 cases of TB occurring in pediatric patients while they were taking anti-TNF treatment ${ }^{6}$. Herein, we report the first fatal case of TBM in a child taking anti-TNF therapy; the child's cerebrospinal fluid (CSF) sample tested negative for Mycobacterium tuberculosis on polymerasechain reaction (PCR), was smear negative for acid-fast bacilli (AFB), and had a low level of adenosine deaminase (ADA).

Corresponding author: Dr. Zumrut Sahbudak Bal.

e-mail: z.sahbudak@gmail.com

Received 4 November 2016

Accepted 20 June 2017

\section{CASE REPORT}

A 13-year-old boy was transferred to our hospital with a suspected diagnosis of tuberculous enteritis and Crohn's disease. $\mathrm{He}$ had been diagnosed with juvenile idiopathic arthritis at 3 years of age and had received oral prednisolone and oral and subcutaneous methotrexate for a period of 3 years. At 6 years of age, the patient was switched to etanercept because of persistent disease; he received etanercept and prednisolone for 7 years. At 12 years of age, the patient was switched to adalimumab due to resistant fever and elevated concentrations of acute-phase reactants. Adalimumab and steroids were administered for 3 months, but his general condition failed to improve. Based on his clinician's decision, the patient received abatacept for 3 months; he then developed pneumonia and a pleural effusion. He was treated with vancomycin and ceftriaxone for 14 days and his general condition improved. He was evaluated for TB; his tuberculin skin test (TST) result was $0 \mathrm{~mm}$ and Quantiferon test result was negative. Two months after having had pneumonia, he developed abdominal pain and underwent surgery for suspected acute appendicitis. Pathologic examination of intestinal lymph nodes revealed granulomatous inflammation. The differential diagnosis included Crohn's disease and tuberculous lymphadenitis. Physical examination revealed no neurologic abnormalities. On the $2^{\text {nd }}$ day of his admission, the patient deteriorated, becoming agitated and displaying features of encephalopathy. Cranial magnetic resonance imaging and venography showed basilar meningeal contrast enhancement and contrast-enhanced nodular lesions (Figure 1 and Figure 2). He was then transferred to the Department of Pediatric Infectious Diseases, at which stage a lumbar puncture was performed. 


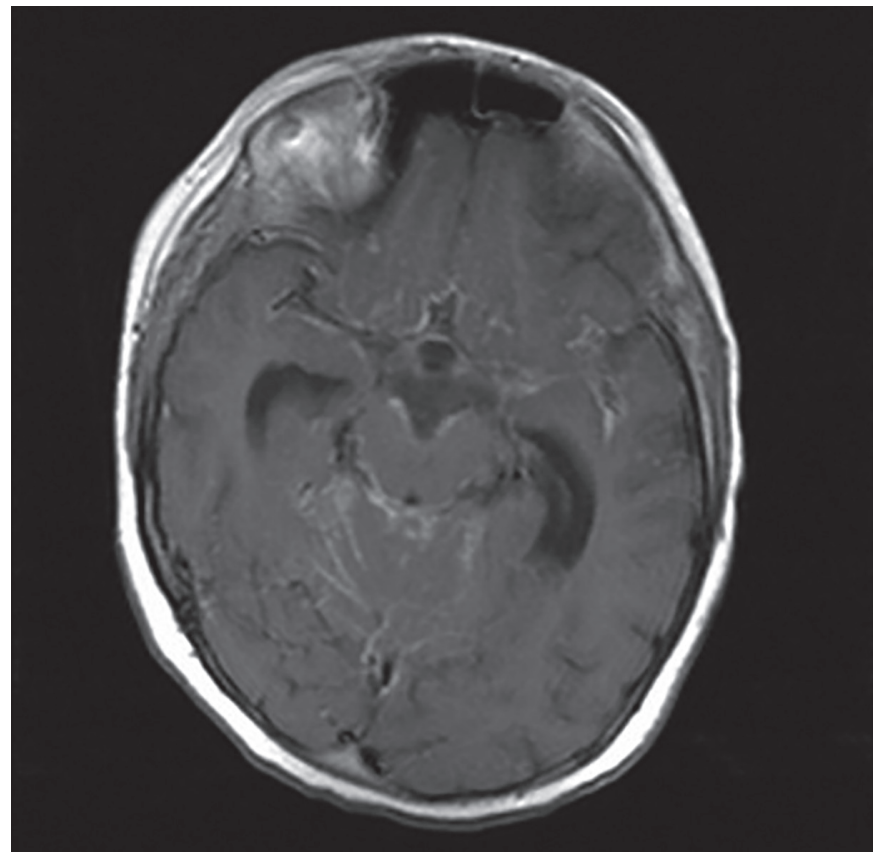

FIGURE 1 - Contrast enhanced T1-weighed magnetic resonance imaging scan showing basilar meningeal enhancement.

Examination of the CSF showed $>1,000$ nucleated cells $(90 \%$ lymphocytes), a protein concentration of $63 \mathrm{mg} / \mathrm{dL}$ (normal range $0-40 \mathrm{mg} / \mathrm{dL}$ ), and a glucose concentration of $51 \mathrm{mg} / \mathrm{dL}$ $(>40 \mathrm{mg} / \mathrm{dL})$. Sputum and CSF samples were negative for M. tuberculosis on PCR testing (Table 1). His serologic status was negative for human immunodeficiency virus, and a lymphocyte panel was normal. Additionally, microscopy of a CSF sample smear revealed no AFB. The ADA level was 1.3IU/L, which was also not suggestive of TBM, but the clinical findings in patients receiving anti-TNF therapy can be challenging.

On the $5^{\text {th }}$ day, the patient developed a right-sided hemiplegia and refractory status epilepticus, and required mechanical ventilation. He required placement of a ventriculo-peritoneal shunt to treat hydrocephalus. His family was asked to bring his specimens from the previous hospital. Histologic re-evaluation of the intestinal lymph node demonstrated granulomatous inflammation with caseation in a small portion of the specimen. Isoniazid, rifampin, ethambutol, pyrazinamide, and streptomycin were started on the $10^{\text {th }}$ day of admission. A lumbar puncture was

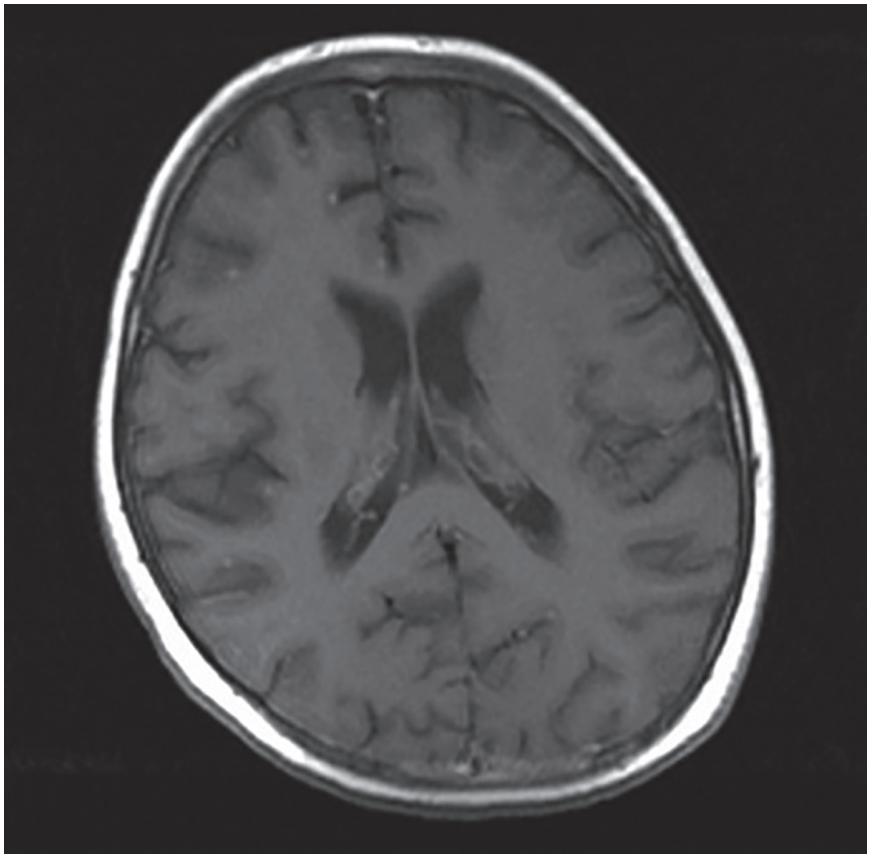

FIGURE 2 - Contrast enhanced T1-weighed magnetic resonance imaging scan showing nodular parenchymal lesions.

repeated; the findings are summarized in Table 1 . The patient died on the $21^{\text {st }}$ day of intensive care unit admission, despite administration of appropriate anti-TB treatment. Six weeks after admission, culture of the CSF revealed M. tuberculosis that was susceptible to all anti-TB drugs.

\section{DISCUSSION}

Tumor necrosis factor is a key cytokine in the host's protective response to $M$. tuberculosis and is important in the development and maintenance of granulomata, which compartmentalize tubercle bacilli during infection and recovery. The consequent breakdown of granulomata with anti-TNF therapy results in reactivation and dissemination of the bacilli, leading to extra-pulmonary disease. TBM develops most commonly as a complication of post-primary infection in infants and young children and from chronic bacteremia in older adults with immune deficiency caused by human immunodeficiency virus infection or drugs, including anti-TNF agents. In our patient, the first mechanism seems more likely given the history

TABLE 1

Cerebrospinal fluid findings of the patient.

\begin{tabular}{llccl}
\hline & CSF WBC count $\left(/ \mathbf{m m}^{3}\right)$, distribution & $\begin{array}{c}\text { CSF protein/ } \\
\text { glucose }\end{array}$ & ADA (IU/L) & Results of PCR/culture for TB \\
\hline Admission & $>1,000(90 \%$ lymphocytes $)$ & $63 / 51$ & 1.3 & Negative/Mycobacterium tuberculosis \\
$10^{\text {th }}$ day & $600(84 \%$ lymphocytes $)$ & $41 / 78$ & - & Negative/Mycobacterium tuberculosis \\
$20^{\text {th }}$ day & $10(50 \%$ lymphocytes $)$ & $150 / 43$ & - & Negative/negative \\
\hline
\end{tabular}

CSF: cerebrospinal fluid; WBC: white blood cell; ADA: adenosine deaminase; IU/L: international unit per liter; PCR: polymerase chain reaction; TB: tuberculosis. 
of pneumonia 2 months before he presented with what was subsequently shown to be TBM. Diagnostic markers of TB, including TST and interferon-gamma release assay (IGRA), were negative at that time; however, the pleural fluid was not examined for TB.

Mortality in patients with TBM is reported to be higher in those with culture-confirmed TB and has been shown to depend on the stage at which anti-TB treatment is initiated ${ }^{1}$. Early recognition is very important, and patients taking TNF antagonists should be followed up closely for the possible development of TB. Although a negative TST or IGRA result cannot eliminate the diagnosis of TB completely, TB screening is important prior to initiating TNF antagonists. Cuomo et al. ${ }^{7}$ reported that 34 (13.6\%) patients whose TB screening tests were negative at baseline displayed conversion of at least 1 screening assay after 12-120 months of treatment with an anti-TNF agent, abatacept, or tocilizumab. On the other hand, conventional tests such as direct examination of CSF, are positive in only $5 \sim 20 \%$ of cases. Moreover, the rate of culture positivity is approximately $40 \%$, and takes approximately 6 weeks. A recent meta-analysis showed that the mean sensitivity and specificity of CSF ADA assays in detecting TMB were 0.79 and 0.91 , respectively ${ }^{8}$.

Our patient received consecutive TNF antagonists and abatacept nearly for 7 years. TNF antagonists and steroids are known to cause false negative TST and IGRA results; hence, we initiated anti-TB drugs immediately, despite the negative AFB, PCR and, IGRA test results. M. tuberculosis was cultured in the patient's CSF specimen 6 weeks later. The rate of TB in patients on adalimumab or infliximab is higher than in those receiving etanercept ${ }^{5,9}$. The median time from the initial use of a TNF antagonist to the diagnosis of TB was 13.4 months for cases exposed to etanercept, 5.5 months for those taking infliximab, and 18.5 months for those on adalimumab ${ }^{5}$. Our patient had a history of concomitant use of etanercept, adalimumab, and abatacept. Etanercept was switched to adalimumab 8 months prior to his current presentation, and he received adalimumab for 3 months. Serious infections have been found to occur more frequently in adalimumab-treated patients than in abatacepttreated and etanercept-treated patients ${ }^{10}$. The patient presented in this report received consecutive biologic agents for nearly 7 years, so it is difficult to single out 1 agent as the facilitating cause of the TB infection. The agent most recently used was abatacept, but abatacept is not considered to increase the risk of developing TB. On the other hand, the patient received adalimumab for 3 months before he developed TB. In a recently published review article, no increased risk of developing TB was found among patients taking abatacept, and pretreatment screening for TB was not recommended ${ }^{11}$. Of 433 abatacepttreated patients, Kremer et al. ${ }^{12}$ reported only 1 possible case of TB - the patient presented with an enlarged lymph node that demonstrated histologic findings compatible with TB.

In conclusion, TBM should be kept in mind in patients receiving biologic agents for rheumatologic diseases, even if the biochemical parameters of the CSF are not suggestive of $\mathrm{TB}$, and if the samples test negative for M. tuberculosis on PCR and negative for acid-fast bacilli on smear microscopy. For patients receiving TNF antagonists, once a provisional diagnosis of TBM is made, empiric treatment must be immediately initiated and must be continued until a negative CSF culture for M. tuberculosis is confirmed.

\section{Conflicts of interest}

The authors declare that there is no conflict of interest.

\section{REFERENCES}

1. Duque-Silva A, Robsky K, Flood J, Barry PM. Risk factors for central nervous system Tuberculosis. Pediatrics. 2015;136(5):e1276-84.

2. Yasui K. Immunity against Mycobacterium tuberculosis and the risk of biologic anti-TNF- $\alpha$ reagents. Pediatr Rheumatol Online J. 2014;12:45. doi: 10.1186/1546-0096-12-45.

3. Gardam MA, Keystone EC, Menzies R, Manners S, Skamene E, Long R, et al. Anti-tumour necrosis factor agents and tuberculosis risk: mechanisms of action and clinical management. Lancet Infect Dis. 2003;3(3):148-55.

4. Wallis RS. Tumour necrosis factor antagonists: structure, function, and tuberculosis risks. Lancet Infect Dis. 2008;8(10):601-11.

5. Dixon WG, Hyrich KL, Watson KD, Lunt M, Galloway J, Ustianowski A, et al. Drug- specific risk of tuberculosis in patients with rheumatoid arthritis treated with anti-TNF therapy: results from the British Society for Rheumatology Biologics Register (BSRBR). Ann Rheum Dis. 2010;69(3):522-8.

6. Toussi SS, Pan N, Walters HM, Walsh TJ. Infections in children and adolescents with juvenile idiopathic arthritis and inflammatory bowel disease treated with tumor necrosis factor- $\alpha$ inhibitors: systematic review of the literature. Clin Infect Dis. 2013;57(9): 1318-30.

7. Cuomo G, D'Abrsca V, Iacono D, Pantano I. The conversion rate of tuberculosis screening tests during biological therapies in patients with rheumatoid arthritis. Clin Rheumatol. 2017;36(2)457-61;

8. Xu HB, Jiang RH, Li L, Sha W, Xiao HP. Diagnostic value of adenosine deaminasein cerebrospinal fluid for tuberculous meningitis: a meta-analysis. Int J Tuberc Lung Dis. 2010;14(11):13827.

9. Tubach F, Salmon D, Ravaud P, Allanore Y, Goupille P, Bréban M,et al. Risk of tuberculosis is higher with anti-tumor necrosis factor monoclonal antibody therapy than with soluble tumor necrosis factor receptor therapy: The three-year prospective French Research Axed on Tolerance of Biotherapies registry. Arthritis Rheum. 2009;60(7):1884-94.

10. Singh JA, Wells GA, Christensen R, Tanjong Ghogomu E, Maxwell L, MacDonald JK, et al. Adverse effects of biologics: a network meta-analysis and Cochrane overview. Cochrane Database Syst Rev. 2011;16(2):CD008794. doi: 10.1002/14651858.CD008794.pub2.

11. Cantini F, Niccoli L, Goletti D. Tuberculosis risk in patients treated with non-anti-tumor necrosis factor- $\alpha$ (TNF- $\alpha$ ) targeted biologics and recently licensed TNF- $\alpha$ inhibitors: data from clinical trials and national registries. J Rheumatol Suppl. 2014;91:56-64.

12. Kremer JM, Genant HK, Moreland LW, Russell AS, Emery P, Abud-Mendoza $\mathrm{C}$, et al. Effects of abatacept in patients with methotrexate-resistant active rheumatoid arthritis: a randomized trial. Ann Intern Med. 2006;144(12):865-76. 